Pacific Journal of Mathematics

FIXED SETS OF INVOLUTIONS

RaNK LEWIS Capobianco 


\title{
FIXED SETS OF INVOLUTIONS
}

\author{
Frank L. Capobianco
}

In their work Differentiable Periodic Maps, Conner and Floyd posed the following question: Given a closed smooth $n$-manifold $M^{n}$, for what values of $k$ does there exist a closed $(n+k)$-manifold $V^{n+k}$ with smooth involution $T$ whose fixed point set is diffeomorphic to $M^{n}$ ? In this paper we show that for many values of $k$ there is a closed manifold with involution $\left(T, V^{n+k}\right)$ whose fixed point set is cobordant to $M^{n}$.

We begin by defining $I_{n}^{k}$ to be the set of classes in the $n$-dimensional unoriented cobordism group $\mathfrak{N}_{n}$ which are represented by an $n$-manifold which is the fixed point set of a closed $(n+k)$-manifold with smooth involution. Some properties of $I_{n}^{k}$ are easy to see-for instance, that $I_{n}^{k}$ is a subgroup of $\mathfrak{R}_{n}$, that $I_{n}^{0}=\mathfrak{N}_{n}$, and that $I_{*}^{k}=\sum_{n=0}^{\infty} I_{n}^{k}$ is an ideal in $\mathfrak{R}_{*}$. It follows from [4] that if the manifold with involution $\left(T, V^{n+1}\right)$ has fixed point set $F^{n}$, then $F^{n}$ bords; hence $I_{n}^{1}=(0)$. It is well-known that if the manifold with involution $\left(T, V^{n+k}\right)$ has fixed point set $F^{n}$, then the mod 2 Euler characteristics $w_{n}\left(F^{n}\right)$ and $w_{n+k}\left(V^{n+k}\right)$ are equal; hence for $k$ odd $I_{n}^{k}$ is contained in $\chi_{n}$, the subgroup of classes in $\mathfrak{R}_{n}$ with zero Euler characteristic.

The main result of this paper is the following:

THEOREM. For $2 \leqq k \leqq n$ and $k$ even, $I_{n}^{k}=\mathfrak{N}_{n} ;$ for $2<k \leqq n$ and $k$ $\operatorname{odd}, I_{n}^{k}=\chi_{n}$.

To prove this result we first verify that $I_{n}^{k}$ is as claimed for $k=2,3$ and that $I_{n}^{k}$ contains an indecomposable cobordism class for each $n$ not of the form $2^{r}-1$ and each $k$ such that $4 \leqq k \leqq n$. Once these facts are established, the theorem itself follows easily by induction.

It is tempting to conjecture that $I_{n}^{k}=(0)$ for $k>n$. In fact, the techniques employed in Section 2 of this paper originally appeared in a dissertation written under the suprevision of R. E. Stong at the University of Virginia which verified this conjecture for $n \leqq 5$. In this regard, the author wishes to express his gratitude and indebtedness to Professor Stong for the generous advice which underlies most of this work.

2. The structure of $I_{n}^{2}$. Because a smooth involution on a closed manifold can not fix an odd number of points, $I_{0}^{k}=(0)$ for $k>0$. In this section we shall prove that for $n>0, I_{n}^{2}=\mathfrak{N}_{n}$, by using the 
Boardman homomorphism $J^{\prime}$ introduced in [1]. In what follows we adopt the notation and terminology of [4].

Let $\mathcal{M}_{m}=\sum_{j=0}^{m} \mathfrak{N}_{\text {, }}(\mathrm{BO}(m-j))$. We define a map $J^{\prime}: \mathscr{M}_{*} \rightarrow \mathfrak{N}_{*}[[\theta]]$, where $\mathfrak{R}_{*}[[\theta]]$ denotes the ring of formal power series in one variable, as follows: If $x$ is an element of $\mathcal{M}_{m}$, set $J_{n}^{\prime}(x)=\Delta^{m} J I^{n+1}(x)$. As an element of $\mathfrak{N}_{n}\left(\mathbf{Z}_{2}\right), J_{n}^{\prime}(x)$ may be written as a sum $\sum_{i=0}^{n} \beta_{i}\left[A, S^{n-t}\right]$ for a unique choice of classes $\beta_{1} \in \mathfrak{N}_{1}$. We define $J^{\prime}(x)=\sum_{i=0}^{\infty} \beta_{\imath} \theta^{\prime}$. Arguments similar to those found in [3] prove that $J^{\prime}$ is a homomorphism of rings.

LEMMA 2.1. Let $\bigcup_{j=0}^{m} \nu^{m-\jmath} \rightarrow F^{\prime}$ be a disjoint union of $(m-j)$ plane bundles. Let $\beta$ be an element of $\mathfrak{N}_{m}$. There exists a manifold with involution $\left(T, V^{m}\right)$ such that $\beta$ is the class of $V^{m}$ and $\bigcup_{j=0}^{m} \nu^{m-1} \rightarrow F^{\prime}$ is the normal bundle to the fixed point set of $\left(T, V^{m}\right)$ if and only if $J^{\prime}\left(\sum_{j=0}^{m}\left[\nu^{m-1} \rightarrow F^{\prime}\right]\right)=\beta \theta^{m}+$ higher power terms.

Proof. Without loss of generality we may suppress the fact we are considering a disjoint union of bundles. Assume $\left(T, V^{m}\right)$ fixes $\nu^{m-J} \rightarrow F^{\prime}$. Then $J_{m}^{\prime}\left(\left[\nu^{m-J} \rightarrow F^{\prime}\right]\right)=\Delta^{m} J^{m+1}\left(\left[\nu^{m-J} \rightarrow F^{\prime}\right]\right)=\left[V^{m}\right]\left[A, S^{0}\right]$ by [4]; so $J^{\prime}\left(\left[\nu^{m-1} \rightarrow F^{\prime}\right]\right)=\left[V^{m}\right] \theta^{m}+$ higher power terms. Assume $J^{\prime}\left(\left[\nu^{m-l} \rightarrow F^{\prime}\right]\right)=\beta \theta^{m}+$ higher power terms. By definition,

$$
0=J_{m-1}^{\prime}\left(\left[\nu^{m-\jmath} \rightarrow F^{\prime}\right]\right)=\Delta^{m} J I^{m}\left(\left[\nu^{m-\jmath} \rightarrow F^{\prime}\right]\right)=\left[A, S\left(\nu^{m-\jmath}\right)\right] .
$$

Suppose $\left(A, S\left(\nu^{m-\jmath}\right)\right)$ bounds $\left(S, M^{m}\right)$. Let

$$
V^{m}=\left(D\left(\nu^{m-\jmath}\right) \cup M^{m}\right) /\left(S\left(\nu^{m-\jmath}\right) \equiv \partial M^{m}\right)
$$

and $T=A \cup S$. The normal bundle to the fixed point set of $\left(T, V^{m}\right)$ is $\nu^{m-J} \rightarrow F^{\prime}$ and hence $\beta=\left[V^{m}\right]$.

We use Lemma 2.1 to explicitly compute $J^{\prime}$ on a basis for $\mu_{*}$ : Let $T$ be the involution on $\mathbf{R} P(n+1)$ defined by mapping $\left[x_{0}, \cdots, x_{n+1}\right]$ to $\left[-x_{0}, x_{1}, \cdots, x_{n+1}\right]$. The normal bundle to the fixed point set of $(T, \mathbf{R} P(n+1))$ is $\mathbf{R}^{n+1} \rightarrow \mathbf{R} P(0) \cup \lambda \rightarrow \mathbf{R} P(n)$, where $\lambda$ is the canonical line bundle. Let $\lambda_{n}$ denote the cobordism class of $\lambda \rightarrow \mathbf{R} P(n)$. Then by $2.1, J^{\prime}\left(\lambda_{n}\right)=1+\sum_{i=0}^{\infty}[V(n+1, i)] \theta^{n+i+1}$, where the $V(n+1, i)$ are the manifolds studied in [5]. In particular, $[V(n+1,0)]=[\mathbf{R} P(n+1)]$ and

$$
\begin{aligned}
{[V(n+1, i)]=} & {[\mathbf{R} P(n+i+1)]+\left[\mathbf{R} P\left(\lambda \oplus \mathbf{R}^{i+1}\right)\right] } \\
& +\sum_{k=0}^{i-1}[\mathbf{R} P(i-k)][V(n+1, k)],
\end{aligned}
$$

where $\mathbf{R} P\left(\lambda \oplus \mathbf{R}^{1+1}\right)$ is used here to denote the total space of the projective space bundle associated to $\lambda \oplus \mathbf{R}^{i+1} \rightarrow \mathbf{R} P(n)$. 
LeMmA 2.2. Let $\alpha \in \mathfrak{N}_{n}$. $\quad \alpha$ belongs to $I_{n}^{2}$ if and only if there exists a 2-plane bundle $\nu^{2} \rightarrow F^{n}$ such that $\alpha=\left[F^{n}\right]$ and the first nonzero term appearing in the power series expansion of $J^{\prime}\left(\left[\nu^{2} \rightarrow F^{n}\right]\right)$ is at least $(n+1)$-dimensional.

Proof. Lemma 2.1 implies that $\alpha$ belongs to $I_{n}^{2}$ if and only if there exists a 2-plane bundle $\nu^{2} \rightarrow F^{n}$ such that $\alpha=\left[F^{n}\right]$ and the first nonzero term appearing in the power series expansion of $J^{\prime}\left(\left[\nu^{2} \rightarrow F^{n}\right]\right)$ is at least $(n+2)$-dimensional. By $[5 ; 2.1], J\left(\mathfrak{N}_{n}(\mathrm{BO}(2))\right) \subset \mathfrak{N}_{n+1}\left(\mathbf{Z}_{2}\right)$. Thus, requiring that the first nonzero term be at least $(n+1)$-dimensional is sufficient.

\section{LEMMA 2.3. $I_{n}^{2}=\mathfrak{N}_{n}$ for $n \geqq 1$.}

Proof. We use Lemma 2.2 to show that for each positive dimension $n$, not of the form $2^{r}-1, I_{n}^{2}$ contains an indecomposable cobordism class; the result then follows from [7]. Because conjugation on $\mathbf{C P}(2)$ fixes $\mathbf{R} P(2), I_{2}^{2}$ contains the class of $\mathbf{R} P(2)$. Because $J^{\prime}\left(\lambda_{4 n+2} \lambda_{0}+\lambda_{2 n+1}^{2}\right)=$ $\left([V(4 n+3,1)]+[V(2 n+2,0)]^{2}\right) \theta^{4 n+4}+$ higher power terms, $I_{4_{n}+2}^{2}$ contains the class of $\mathbf{R} P(4 n+2)$. Because $J^{\prime}\left(\lambda_{4 n} \lambda_{0}+\lambda_{2 n}^{2}\right)=$ $[V(4 n+1,1)] \theta^{4 n+2}+$ higher power terms, $I_{4 n}^{2}$ contains the class of $\mathbf{R} P(4 n) \cup \mathbf{R} P(2 n) \times \mathbf{R} P(2 n)$. Suppose $n=2^{p}(2 q+1)-1$ for $p, q>$ 0 . For each $j, 0 \leqq j \leqq n$, let the cobordism class $\gamma_{j}$ be defined by

$$
\gamma_{1}=\left\{\begin{array}{lc}
1 & j=0 \\
0 & 1 \leqq j \leqq 2^{p}+1 \\
{\left[V\left(2^{p}+1, j-2^{p}-1\right)\right]} & 2^{p}+2 \leqq j \leqq 2^{p+1} q-1 \\
{\left[V\left(2^{p}+1, j-2^{p}-1\right)\right]+\left[V\left(2^{p+1} q, j-2^{p+1} q\right)\right]} & 2^{p+1} q \leqq j \leqq n .
\end{array}\right.
$$

Let $\gamma=\sum_{j=0}^{n} \gamma_{j} \lambda_{n-1} \lambda_{0}$. Then $J^{\prime}\left(\lambda_{2}^{p} \lambda_{2}^{p+1} q^{p-1}+\gamma\right)=\beta \theta^{n+1}+$ higher power terms, for some class $\beta \in \mathfrak{N}_{n+1}$. By Lemma 2.2, the base of $\lambda_{2}^{p} \lambda_{2}^{p+1}{ }_{q-1}^{p}+\gamma$ belongs to $I_{n}^{2}$; by $[5 ; 4.2]$ and $[6 ; 3.4]$, this class is indecomposable.

3. The structure of $I_{n}^{k}, 2<k \leqq n$. Let $\xi^{k} \rightarrow M^{n-k+1}$ be an arbitrary $k$-plane bundle and let $\mathbf{R} P\left(\xi^{k}\right)$ denote the total space of the associated projective space bundle.

LEMMA 3.1. $I_{n}^{k}$ contains the cobordism class of $\mathbf{R} P\left(\xi^{k}\right) \cup M^{n-k+1} \times$ $\mathbf{R} P(k-1)$. 
Proof. Consider the Whitney sum $\xi^{k} \oplus \mathbf{R}^{k} \rightarrow M^{n-k+1}$ and the total space $\mathbf{R} P\left(\xi^{k} \oplus \mathbf{R}^{k}\right)$ of the associated projective space bundle. Multiplication by -1 in the fibers of $\xi^{k}$ induces an involution on $\mathbf{R} P\left(\xi^{k} \oplus \mathbf{R}^{k}\right)$ whose fixed point set is $\mathbf{R} P\left(\xi^{k}\right) \cup M^{n-k+1} \times \mathbf{R} P(k-1)$.

\section{LEMMA 3.2. $I_{n}^{3}=\chi_{n}$.}

Proof. Recall from $\S 1$ that $I_{*}^{3}$ is contained in $\chi_{*}=\Sigma_{n=0}^{\infty} \chi_{n}$, the ideal of classes in $\mathfrak{N}_{*}$ with zero Euler characteristic. It is not hard to see that $\chi_{n}$ contains an indecomposable cobordism class for each dimension $n \geqq 4, n \neq 2^{r}-1$, and that $\chi_{*}$ is generated by these elements. In [6; 8.1] Stong exhibited for each $n \geqq 4, n \neq 2^{r}-1$, a 3 -plane bundle $\xi^{3} \rightarrow M^{n-2}$ such that the cobordism class of $\mathbf{R} P\left(\xi^{3}\right)$ is indecomposable. Thus by Lemma $3.1 I_{n}^{3}$ contains the indecomposable class $\mathbf{R} P\left(\xi^{3}\right) \cup M^{n-2} \times \mathbf{R} P(2)$, and therefore $I_{*}^{3}=\chi *$.

To prove that $I_{n}^{k}$ is as stated in $\S 1$ we need finally to show that $I_{n}^{k}$ contains an indecomposable cobordism class for each dimension $n$ not of the form $2^{r}-1$ and each $k$ such that $4 \leqq k \leqq n$.

LEMMA 3.3. $I_{n}^{k}$ contains an indecomposable cobordism class for each $n \neq 2^{r}-1$ and each $k$ such that $4 \leqq k \leqq \alpha(n)$, where $\alpha(n)$ denotes the number of ones in the dyadic expansion of $n$.

Proof. Recall the Stong manifolds from [6]: Let $\left(n_{1}, \cdots, n_{k}\right)$ be a partition of $n+k-1$ and let $p: \mathbf{R}\left(P\left(n_{1}, \cdots, n_{k}\right) \rightarrow \mathbf{R} P\left(n_{1}\right) \times \cdots \times \mathbf{R} P\left(n_{k}\right)\right.$ be the projective space bundle associated to $\lambda_{1} \oplus \cdots \oplus \lambda_{k} \rightarrow \mathbf{R} P\left(n_{1}\right) \times$ $\cdots \times \mathbf{R} P\left(n_{k}\right)$, where $\lambda_{t}$ is the pullback of the canonical line bundle over the $i$ th factor. By Lemma $3.1 I_{n}^{k}$ contains the cobordism class of $\mathbf{R} P\left(n_{1}, \cdots, n_{k}\right) \cup \mathbf{R} P\left(n_{1}\right) \times \cdots \times \mathbf{R} P\left(n_{k}\right) \times \mathbf{R} P(k-1)$; and by [6; 3.4] this class is indecomposable if and only if $\left(\begin{array}{c}n-1 \\ n_{1}\end{array}\right)+\cdots+\left(\begin{array}{c}n-1 \\ n_{k}\end{array}\right)=1 \mathrm{mod}$ 2. It suffices then to exhibit for each choice of $n$ and $k$ a partition $\left(n_{1}, \cdots, n_{k}\right)$ of $n-k+1$ such that $\left(\begin{array}{c}n-1 \\ n_{1}\end{array}\right)+\cdots+\left(\begin{array}{c}n-1 \\ n_{k}\end{array}\right)=1 \bmod 2 . \quad$ If $n=2^{r_{1}}+\cdots+2^{r_{t}}, r_{1}>\cdots>r_{t}>0$, and $4 \leqq k \leqq t$, then

$$
\left(2^{r_{1}}+\cdots+2^{r_{t-k+2}}, 2^{r_{t-k+3}}-1, \cdots, 2^{r_{t-1}}-1,2^{r_{1}-1}-1,2^{r_{t}-1}-1\right)
$$

is as required. If $n=2^{r_{1}}+\cdots+2^{r_{1}}$, where $r_{1}>\cdots>r_{t}=0$ and there exists an $i, 2 \leqq i \leqq t$, such that $r_{1-1}>r_{i}+1$, and $4 \leqq k \leqq t$, then

$$
\left(2^{r_{1}}-2,2^{r_{2}}-1, \cdots, 2^{r_{k-2}}-1,2^{r_{k-1}}+\cdots+2^{r_{1-1}}, 1\right)
$$

is as required. 
To prove that $I_{n}^{k}$ contains an indecomposable class for each $n \neq 2^{r}-$ 1 and each $k$ such that $\alpha(n)<k \leqq n$ we must use a different technique, provided by the following:

LEMMA 3.4. If $M^{n}$ is a closed manifold such that $w_{1}\left(M^{n}\right)=0$ for $i>\alpha(n)+1$, then $I_{n}^{k}$ contains the class of $M^{n}$ for $\alpha(n)<k \leqq n$.

Proof. The twist involution on $M^{n} \times M^{n}$ is defined by sending $(x, y)$ to $(y, x)$ and has fixed point set $M^{n}$; furthermore, the normal bundle to $M^{n}$ in $M^{n} \times M^{n}$ is the tangent bundle $\tau M^{n} \rightarrow M^{n}$. By Lemma 2.1 $J^{\prime}\left(\left[\tau M^{n} \rightarrow M^{n}\right]\right)=\left[M^{n} \times M^{n}\right] \theta^{2 n}+$ higher power terms. By [4], since $w_{i}\left(M^{n}\right)=0$ for $i>\alpha(n)+1$ there exists an $(\alpha(n)+1)$-plane bundle $\xi \rightarrow N^{n}$ such that $\xi \oplus \mathbf{R}^{n-\alpha(n)-1} \rightarrow N^{n}$ is cobordant to $\tau M^{n} \rightarrow M^{n}$. Therefore, $J^{\prime}\left(\left[\xi \rightarrow N^{n}\right]\right)=J^{\prime}\left(\left[\tau M^{n} \rightarrow M^{n}\right]\right)=$ $\left[M^{n} \times M^{n}\right] \theta^{2 n}+$ higher power terms. By Lemma 2.1, for each $k$ such that $\alpha(n)<k \leqq n$ there exists a manifold with involution $\left(T, V^{n+k}\right)$ such that the normal bundle to the fixed point set of $T$ is $\xi \bigoplus \mathbf{R}^{k-\alpha(n)-1} \rightarrow N^{n}$. Therefore the cobordism class of $M^{n}$, which is the same as that of $N^{n}$, belongs to $I_{n}^{k}$ for $\alpha(n)<k \leqq n$.

It remains then to show that for each dimension $n \neq 2^{r}-1$ there is an indecomposable manifold $M^{n}$ such that $w_{i}\left(M^{n}\right)=0$ for $i>$ $\alpha(n)+1$. For this purpose we define generalized Stong manifolds as follows: Let $N=\left(N_{1}, \cdots, N_{k}\right)$ be a $k$-tuple where for each $i, 1 \leqq i \leqq k, N_{i}$ is a $t_{t}$-tuple $\left(n_{t 1}, \cdots, n_{t_{t}}\right)$ of nonnegative integers. Define $\mathbf{R} P\left(N_{1}, \cdots, N_{k}\right)$ to be the total space of the projective space bundle associated to $\lambda_{1} \oplus \cdots \oplus \lambda_{k} \rightarrow \mathbf{R} P\left(N_{1}\right) \times \cdots \times \mathbf{R} P\left(N_{k}\right)$, where $\lambda_{1}$ is the pullback of the canonical line bundle over the Strong manifold $\mathbf{R} P\left(N_{1}\right)$. Letting $\left|N_{1}\right|$ denote $n_{11}+\cdots+n_{t_{t}}+t_{1}-1$ and $|N|=$ $\left|N_{1}\right|+\cdots+\left|N_{k}\right|+k-1$, we see that $\mathbf{R} P\left(N_{1}, \cdots, N_{k}\right)$ is an $|N|-$ dimensional manifold.

LEMMA 3.5. $\mathbf{R} P\left(N_{1}, \cdots, N_{k}\right)$ represents an indecomposable cobordism class if and only if

$$
\left(\begin{array}{c}
|N|-1 \\
\left|N_{1}\right|
\end{array}\right)+\cdots+\left(\begin{array}{c}
|N|-1 \\
\left|N_{k}\right|
\end{array}\right) \text { is odd }
$$

Proof. There is a degree one map $\mathbf{R} P\left(N_{1}\right) \times \cdots \times \mathbf{R} P\left(N_{k}\right) \rightarrow$ $\mathbf{R} P\left(\left|N_{1}\right|\right) \times \cdots \times \mathbf{R} P\left(\left|N_{k}\right|\right)$ such that the pullback of $\lambda_{1} \oplus \cdots \oplus \lambda_{k} \rightarrow \mathbf{R} P\left(\left|N_{1}\right|\right) \times \cdots \times \mathbf{R} P\left(\left|N_{k}\right|\right)$ is $\lambda_{1} \oplus \cdots \oplus \lambda_{k} \rightarrow \mathbf{R} P\left(N_{1}\right) \times$ $\cdots \times \mathbf{R} P\left(N_{k}\right)$. By [6;2.4], $\mathbf{R} P\left(N_{1}, \cdots, N_{k}\right)$ is indecomposable if and only if $\mathbf{R} P\left(\left|N_{1}\right|, \cdots,\left|N_{k}\right|\right)$ is; but, by $[\mathbf{6} ; 3.4] \mathbf{R} P\left(\left|N_{1}\right|, \cdots,\left|N_{k}\right|\right)$ is indecomposable if and only if 


$$
\left(\begin{array}{c}
|N|-1 \\
\left|N_{1}\right|
\end{array}\right)+\cdots+\left(\begin{array}{c}
|N|-1 \\
\left|N_{k}\right|
\end{array}\right) \text { is odd }
$$

The cohomology and Stiefel-Whitney classes of $\mathbf{R} P\left(N_{1}, \cdots, N_{k}\right)$ are explicitly computable from [4]. In fact, let $H^{*}\left(\mathbf{R} P\left(n_{y y}\right) ; \mathbf{Z}_{2}\right)=$ $\mathbf{Z}_{2}\left[\alpha_{y}\right] /\left(\alpha_{y^{y}}^{n_{y}+1}=0\right)$ and $c_{1}$ and $e$ represent the characteristic class of the canonical line bundle over $\mathbf{R} P\left(N_{\imath}\right)$ and $\mathbf{R} P\left(N_{1}, \cdots, N_{k}\right)$ respectively. Suppressing all bundle maps, we may write

$$
w\left(\mathbf{R} P\left(N_{1}, \cdots, N_{k}\right)\right)=\prod_{i=1}^{k} \prod_{j=1}^{t_{i}}\left(1+\alpha_{i y}\right)^{n_{i}+1}\left(1+c_{i}+\alpha_{i j}\right)\left(1+e+c_{1}\right) .
$$

LEMMA 3.6. For each dimension $n \neq 2^{r}-1$ there is an indecomposable manifold $M^{n}$ such that $w_{1}\left(M^{n}\right)=0$ for $i>\alpha(n)+1$.

Proof. If $n=2^{r_{1}}+\cdots+2^{r_{t}}, \quad r_{1}>\cdots>r_{t}>0, \quad$ let $\quad M^{n}=$ $\mathbf{R} P\left(\left(2^{r_{1}}-1, \cdots, 2^{r_{1-1}}-1,0\right), \quad\left(2^{r_{1}-1}-1\right), \quad\left(2^{r_{1}-1}-1\right)\right)$. If $\quad n=$ $2^{r_{1}}+\cdots+2^{r_{t}}+2^{t}+2^{t^{-1}}+\cdots+1, \quad r_{1}>\cdots>r_{t}>j+1, \quad$ let $\quad M^{n}=$ $\mathbf{R} P\left(\left(2^{r_{1}}-1, \cdots, 2^{r_{1-1}}-1,2^{r_{1}-1}-1,0\right),\left(2^{r_{1}-1}-1\right),\left(2^{l}-1\right), \cdots,\left(2^{0}-1\right)\right)$. That these manifolds are indecomposable is a direct consequence of Lemma 3.5. That $w_{l}\left(M^{n}\right)=0$ for $i>\alpha(n)+1$ is immediate from the given expansion of $w\left(\mathbf{R} P\left(N_{1}, \cdots, N_{k}\right)\right)$ taken with the fact that multiplication in $H^{*}\left(\mathbf{R} P\left(N_{1}, \cdots, N_{k}\right) ; \mathbf{Z}_{2}\right)$ is subject to the relations $\Pi_{j=1}^{t_{i}}\left(c_{t}+\alpha_{y}\right)=0$ for each $i, 1 \leqq i \leqq k$, and $\Pi_{t=1}^{k}\left(e+c_{i}\right)=0$.

Let now assemble the above lemmas to prove:

THEOREM. For $2 \leqq k \leqq n$ and $k$ even, $I_{n}^{k}=\mathfrak{R}_{n} ;$ for $2<k \leqq n$ and $k$ $\operatorname{odd}, I_{n}^{k}=\chi_{n}$.

Proof. Let $4 \leqq k \leqq n$ and assume inductively that for $2 \leqq j<k \leqq n$ and $j$ even, $I_{n}^{\prime}=\mathfrak{N}_{n}$, while for $2<j<k \leqq n$ and $j$ odd, $I_{n}^{\prime}=\chi_{n}$. We must show that $I_{n}^{k}$ is as claimed. Let $\alpha \in \mathfrak{N}_{n}$, with $w_{n}(\alpha)=0$ if $k$ is odd. If $\alpha$ is decomposable, say $\alpha=\beta \gamma$ where $\beta \in \mathfrak{N}_{p}$ and $\gamma \in \mathfrak{N}_{q}$ with $w_{q}(\gamma)=0$ if $k$ is odd, then by induction $\beta \in I_{p}^{2}$ and $\gamma \in I_{q}^{k-2}$. Clearly $I_{p}^{2} I_{q}^{k-2} \subset I_{n}^{k}$, so $\alpha \in I_{n}^{k}$. If $\alpha$ is indecomposable, then by Lemmas 3.3-3.6 $\alpha$ belongs to $I_{n}^{k} \bmod$ decomposables; but, since $I_{n}^{k}$ contains all decomposables, $\alpha \in I_{n}^{k}$.

\section{REFERENCES}

1. J. M. Boardman, On manifolds with involution, Bull. Amer. Math. Soc., 73 (1967), 136-138.

2. F. L. Capobianco, Involutions and semi-free $S^{\prime}$-actions, Thesis, University of Virginia, 1975. 
3. P. E. Conner, Seminar on periodic maps, Lecture Notes in Math. No. 46, Springer, New York, 1967.

4. P. E. Conner and E. E. Floyd, Differentiable Periodic Maps, Springer, Berlin, 1964.

5. P. E. Conner and E. E. Floyd, Fibring within a cobordism class, Michigan Math. J., 12 (1965), 33-47.

6. R. E. Stong, On fibering of cobordism classes, Trans. Amer. Math. Soc., 178 (1973), 431-447.

7. R. Thom, Quelques proprietes globales des varietes differentiables, Comm. Math. Helv., 28 (1954), 17-86.

Received December 7, 1976. This research was supported, in part, by the National Science Foundation, Grant MCS 76-06373.

UNIVERSITY OF WISCONSIN

MADISON, WI 53706 



\section{PACIFIC JOURNAL OF MATHEMATICS}

\section{EDITORS}

RICHARD ARENS (Managing Editor)

University of California

Los Angeles, CA 90024

R. A. BeAumont

University of Washington

Seattle, WA 98105

C. C. MOORE

University of California

Berkeley, CA 94720
J. DUGUNDJI

Department of Mathematics

University of Southern California

Los Angeles, CA 90007

R. FINN AND J. MILGRAM

Stanford University

Stanford, CA 94305

\section{ASSOCIATE EDITORS}
E. F. BECKENBACH
B. H. NEUMANN
F. WOLF
K. YoshidA

\section{SUPPORTING INSTITUTIONS}

UNIVERSITY OF BRITISH COLUMBIA

UNIVERSITY OF SOUTHERN CALIFORNIA

CALIFORNIA INSTITUTE OF TECHNOLOGY

STANFORD UNIVERSITY

UNIVERSITY OF CALIFORNIA

UNIVERSITY OF HAWAII

MONTANA STATE UNIVERSITY

UNIVERSITY OF TOKYO

UNIVERSITY OF NEVADA

UNIVERSITY OF UTAH

NEW MEXICO STATE UNIVERSITY

OREGON STATE UNIVERSITY

UNIVERSITY OF OREGON

OSAKA UNIVERSITY

WASHINGTON STATE UNIVERSITY

UNIVERSITY OF WASHINGTON

AMERICAN MATHEMATICAL SOCIETY

The Supporting Institutions listed above contribute to the cost of publication of this Journal, but they are not owners or publishers and have no responsibility for its contents or policies.

Mathematical papers intended for publication in the Pacific Journal of Mathematics should be in typed form or offset-reproduced (not dittoed), double spaced with large margins. Underline Greek letters in red, German in green, and script in blue. The first paragraph or two must be capable of being used separately as a synopsis of the entire paper. Items of the bibliography should not be cited there unless absolutely necessary, in which case they must be identified by author and Journal, rather than by item number. Manuscripts, in duplicate, may be sent to any one of the four editors. Please classify according to the scheme of Math. Reviews, Index to Vol. 39. All other communications should be addressed to the managing editor, or Elaine Barth, University of California, Los Angeles, California, 90024.

100 reprints are provided free for each article, only if page charges have been substantially paid. Additional copies may be obtained at cost in multiples of 50 .

The Pacific Journal of Mathematics is issued monthly as of January 1966. Regular subscription rate: $\$ 72.00$ a year (6 Vols., 12 issues). Special rate: $\$ 36.00$ a year to individual members of supporting institutions.

Subscriptions, orders for numbers issued in the last three calendar years, and changes of address should be sent to Pacific Journal of Mathematics, 103 Highland Boulevard, Berkeley, California, 94708.

PUBLISHED BY PACIFIC JOURNAL OF MATHEMATICS, A NON-PROFIT CORPORATION

Printed at Jerusalem Academic Press, POB 2390, Jerusalem, Israel. 


\section{Pacific Journal of Mathematics}

\section{Vol. 75, No. $2 \quad$ October, 1978}

Susan Jane Zimmerman Andima and W. J. Thron, Order-induced

topological properties ................................... 297

Gregory Wade Bell, Cohomology of degree 1 and 2 of the Suzuki groups . . 319

Richard Body and Roy Rene Douglas, Rational homotopy and unique

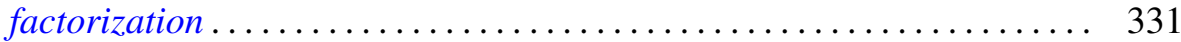

Frank Lewis Capobianco, Fixed sets of involutions ................. 339

L. Carlitz, Some theorems on generalized Dedekind-Rademacher sums .... 347

Mary Rodriguez Embry and Alan Leslie Lambert, The structure of a special class of weighted translation semigroups .....................

Steve Ferry, Strongly regular mappings with compact ANR fibers are

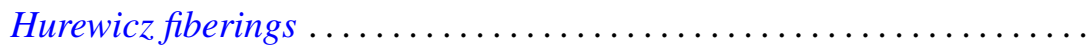

Ivan Filippenko and Marvin David Marcus, On the unitary invariance of the

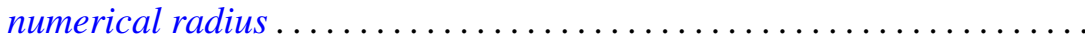

$\mathrm{H}$. Groemer, On the extension of additive functionals on classes of convex

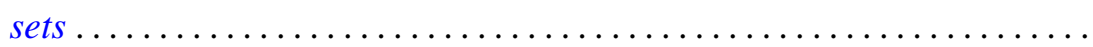

Rita Hall, On the cohomology of Kuga's fiber variety ............... 411

H. B. Hamilton, Congruences on $\mathrm{N}$-semigroups ................. 423

Manfred Herrmann and Rolf Schmidt, Regular sequences and lifting

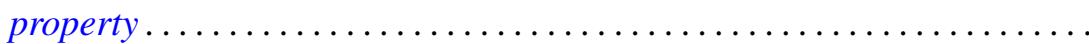

James Edgar Keesling, Decompositions of the Stone-Čech compactification which are shape equivalences .....................

Michael Jay Klass and Lawrence Edward Myers, On stopping rules and the expected supremum of $S_{n} / T_{n}$

Ronald Charles Linton, $\lambda$-large subgroups of $C_{\lambda}$-groups

William Owen Murray, IV and L. Bruce Treybig, Triangulations with the free cell property ............................

Louis Jackson Ratliff, Jr., Polynomial rings and $H_{i}$-local rings ...

Michael Rich, On alternate rings and their attached Jordan rings....

Gary Sampson and H. Tuy, Fourier transforms and their Lipschitz

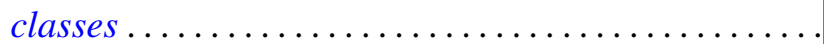

Helga Schirmer, Effluent and noneffluent fixed points on dendrites ...

Daniel Byron Shapiro, Intersections of the space of skew-symmetric maps

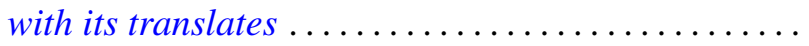

Edwin Spanier, Tautness for Alexander-Spanier cohomology ...

Alan Stein and Ivan Ernest Stux, A mean value theorem for binary digits ...

Franklin D. Tall, Normal subspaces of the density topology . .

William Yslas Vélez, Prime ideal decomposition in $F\left(\mu^{1 / p}\right) \ldots$ 